\title{
Effects of the platelet-activating factor (PAF) on selected quality parameters of cryopreserved bull semen (AI) with reduced sperm motility
}

\author{
M. Lecewicz', W. Kordan'1, A. Majewska1, S. Kamiński², \\ A. Dziekońska ${ }^{1}$, K. Mietelska ${ }^{1}$ \\ ${ }^{1}$ Departament of Animal Biochemistry and Biotechnology, \\ University of Warmia and Mazury in Olsztyn, Oczapowskiego 5, 10-718 Olsztyn, Poland \\ ${ }^{2}$ Departament of Animal Genetics, University of Warmia and Mazury in Olsztyn, \\ Oczapowskiego 5, 10-718 Olsztyn, Poland
}

\begin{abstract}
The aim of the study was to determine the effects of platelet-activating factor (PAF) on selected quality parameters of cryopreserved bull semen with reduced sperm motility used for artificial insemination. The aim of experiment 1 was to identify the optimal concentration of the phospholipid able to preserve sperm viability. Cryopreserved semen was treated with different PAF concentrations: $1 \times 10^{-5} \mathrm{M}, 1 \times 10^{-6} \mathrm{M}, 1 \times 10^{-7} \mathrm{M}, 1 \times 10^{-8} \mathrm{M}$ and $1 \times 10^{-9} \mathrm{M}$. The experiment demonstrated that PAF at concentration $1 \times 10^{-9} \mathrm{M}$ increased most the sperm viability parameters (motility parameters, plasma membrane integrity and mitochondrial function) after $120 \mathrm{~min}$ of incubation of thawed semen at $37^{\circ} \mathrm{C}$. Cryopreserved bull semen with reduced sperm motility (below 70\%) was supplemented with PAF in a concentration of $1 \times 10^{-9} \mathrm{M}$. A statistically significant increase in sperm motility, percentage of linear motile spermatozoa and VSL value was observed after 120 min incubation of sperm with $1 \times 10^{-9} \mathrm{M}$ PAF. Sperm supplementation with PAF also had positive effects on plasma membrane integrity and percentage of spermatozoa with preserved mitochondrial transmembrane potential, but the differences were not statistically significant. The results indicated positive effects of PAF supplementation at a concentration of $1 \times 10^{-9} \mathrm{M}$ on the selected sperm quality parameters in cryopreserved bull semen with reduced motility.
\end{abstract}

Key words: bull, spermatozoa, PAF, viability, cryopreservation. 


\section{Introduction}

Insemination with cryopreserved semen is the most popular practice used in the controlled reproduction of cattle. The main factor determining the success of the insemination procedure is a semen quality (Walsh et al. 2011). Sperm motility is the basic parameter of semen quality analysed at insemination centres. Many researchers have suggested that the percentage of motile spermatozoa is an indicator of plasma membrane integrity and metabolic activity of semen (Johnson et al. 2000, Estienne et al. 2007, Kaeoket et al. 2010). For effective insemination, the cryopreserved semen has to be of a high biological quality.

The platelet-activating factor (1-0-alkyl-2-acetylsn-glycerol-3-phosphoryl-choline) (PAF) is a specific signalling phospholipid that has pleiotropic biological properties in addition to platelet activation (Braquet et al. 1987, Roudebush and Diehl 2001). This naturally occurring, acetylated membrane glycerophospholipid plays an important role in the reproductive process, both in males and females (Kordan et al. 2003).

The synthesis of PAF from membrane phospholipids in various types of activated cells, mainly basophiles, neutrophils, monocytes, macrophages and endothelial cells, is catalysed by phospholipase A2 (Roudebush et al. 2002). PAF plays an important role in mammalian reproduction, affecting, for example, the ovulation and fertilization process. In addition, it is involved in the pre-implantation development of the embryo and its subsequent implantation (Harper 1989, Roudebush et al. 2002).

PAF is present in human spermatozoa (Minhas et al. 1991), and in other primates, including squirrel monkey (Roudebush and Mathur 1998), macaque (Diaz et al. 1999) and rhesus monkey (Roudebush et al. 2002), and in domestic animals such as rabbit, rooster, boar, bull and stallion (Kumar et al. 1988, Hough and Parks 1994, Roudebush and Diehl 2001). Many studies carried out in various animal species and in humans have demonstrated the positive effect of supplementation with exogenous PAF on sperm motility, plasma membrane integrity, capacitation and the acrosome reaction of semen preserved either in liquid form or cryopreserved (Aravindakshan and Sharma 1996, Roudebush et al. 2002, Odeh et al. 2003, Kordan and Strzeżek 2006, Kordan et al. 2009, Kheradmand et al. 2009, Kordan et al. 2010, Esmaeilpour et al. 2014).

The aim of the study was to determine the effects of PAF on selected quality parameters of cryopreserved bull semen with reduced sperm motility.

\section{Materials and Methods}

\section{Experiment 1}

The experimental material was cryopreserved semen obtained from 10 bulls reared at the Animal Breeding and Insemination Centre in Bydgoszcz, used for insemination practice. Cryopreserved spermatozoa $\left(20 \times 10^{6}\right.$ spermatozoa/ $\left./ \mathrm{cm}^{3}\right)$ were treated with different concentrations of PAF: $1 \times 10^{-5} \mathrm{M}, 1 \times 10^{-6} \mathrm{M}$, $1 \times 10^{-7} \mathrm{M}, 1 \times 10^{-8} \mathrm{M}$ and $1 \times 10^{-9} \mathrm{M}$ at $37^{\circ} \mathrm{C}$. Cryopreserved semen without PAF supplementation was used as the control. Sperm viability (motility parameters, plasma membrane integrity and mitochondrial function) was examined at different time intervals: 0, 30, 60, 90 and $120 \mathrm{~min}$ for each PAF treatment, together with the control (without PAF).

\section{Experiment 2}

The experimental material was cryopreserved semen with reduced sperm motility obtained from 10 bulls reared at the Animal Breeding and Insemination Centre in Bydgoszcz, used for insemination practice. PAF was used at a concentration of $10^{-9} \mathrm{M}$. The assessments of the quality parameters of the cryopreserved bull semen and sample incubation times were identical to those in the experiment 1.

\section{Sperm viability assessments}

\section{Motility}

Sperm motility was evaluated using the computer assisted sperm analysis (CASA) system (VideoTesT Sperm 2.1, St. Petersburg, Russia). Aliquots of sperm samples were placed in a Makler Chamber and examined at $37^{\circ} \mathrm{C}$ under a phase-contrast microscopy system coupled to a video camera adapted to the VideoTesT Sperm system. All motility parameters were analysed in accordance with the recommendations given by the World Health Organization (Elia et al. 2010). The parameters analysed included total sperm motility (\%), linear motile spermatozoa (\%), nonlinear motile spermatozoa (\%) and VSL (Straight Line Velocity).

\section{Plasma membrane integrity}

Sperm plasma membrane integrity was assessed using dual fluorescent staining, SYBR-14 and PI (Live/Dead Sperm Viability Kit; Molecular Probes), 
as described by Garner and Johnson (1995), with slight modifications. Briefly, aliquots of sperm samples $\left(20 \times 10^{6}\right.$ spermatozoa $\left./ \mathrm{cm}^{3}\right)$ were incubated with SYBR-14 (1mM SYBR-14 in DMSO) and PI solutions $(2.4 \mu \mathrm{M}$ PI in Tyrode's salt solution) for 10 minutes at $37^{\circ} \mathrm{C}$. Following incubation, stained sperm cells were placed on microscopic slides and examined at $600 \mathrm{x}$ magnification under a fluorescence microscope (Olympus CH 30 RF-200, Tokyo, Japan). Only sperm cells displaying only bright green fluorescence were considered viable spermatozoa with an undamaged plasma membrane. A minimum of 200 cells per slide were examined in random fields of each aliquot.

\section{Mitochondrial function assessed by fluorescent microscopy}

The sperm mitochondrial function was assessed using dual staining with fluorescent probes, 5,5',6,6'-tetrachloro-1,1',3,3'-tetraethylbenzimidazolylcarbocyanine iodide, JC-1 (Molecular Probes, Eugene, USA) with propidium iodide (PI, Sigma Chemical Co., St. Louis, MO, USA), according to a previously described method (Thomas et al. 1998), with some modifications (Dziekońska et al. 2009). Aliquots of sperm samples $\left(20 \times 10^{6}\right.$ spermatozoa $\left./ \mathrm{cm}^{3}\right)$ were incubated with JC-1 solution (1mg JC- $1 / \mathrm{cm}^{3}$ dimethylsulfoxide, DMSO) for $15 \mathrm{~min}$ at $37^{\circ} \mathrm{C}$. Following incubation, sperm samples were stained with PI $(10 \mu \mathrm{l}$ of PI solution in $0.5 \mathrm{mg} / \mathrm{cm}^{3}$ phosphate buffered solution) for 10 minutes at $37^{\circ} \mathrm{C}$, washed $(600 \mathrm{x} \mathrm{g}, 5 \mathrm{~min}$ at room temperature) and the sperm pellets were re-suspended in a HEPES buffered solution $(10 \mathrm{mM}$ HEPES, $0.85 \% \mathrm{NaCl}, 0.1 \%$ bovine serum albumin, $\mathrm{pH}$ 7.4). Stained sperm samples were placed on microscopic slides, covered with coverslips $(22 \times 22$ $\mathrm{mm}$ ) and examined under a fluorescence microscope (Olympus CH 30 RF-200). Viable spermatozoa with functional mitochondria emitted orange-red fluorescence. Two slides were evaluated per sample and 200 spermatozoa were counted per slide.

\section{Statistical analysis}

Values were expressed as the mean + standard deviation (SD). The data were analysed by ANOVA, followed by the Duncan multiple comparison test (Experiment I), and by the t-Student test (Experiment 2), using the Statistica software package (StatSoft Incorporation, Tulsa OK., USA). Differences between means were considered significant at $\mathrm{p} \leq 0.05$.

\section{Results}

\section{Experiment 1}

Changes in the percentage of motile spermatozoa are presented in Fig. 1A. A higher percentage of motile spermatozoa was found from the first minutes of incubation of samples with different concentrations of exogenous PAF when compared to the control. This trend continued throughout the entire incubation period. Statistically significant differences at $\mathrm{p} \leq 0.05$ were found starting from minute 30 of the experiment. The highest percentage of motile sperm was found for PAF at a concentration of $1 \times 10^{-9} \mathrm{M}$.

Regardless of PAF concentration and incubation time, the supplementation of samples with the phospholipid had a positive effect on all sperm kinematic parameters, i.e. VCL, VAP, ALH, BCF (data not shown) and VSL (Fig. 1B). In minute 90 of the experiment the supplementation with PAF at concentrations $1 \times 10^{-8} \mathrm{M}$ and $1 \times 10^{-9} \mathrm{M}$ caused a statistically significant increase in the linear motility of spermatozoa as compared to the control. In minute 120 statistically significant differences were found between the control and samples treated with PAF at concentrations of $1 \times 10^{-6} \mathrm{M}$ and $1 \times 10^{-9} \mathrm{M}$.

The effect of different PAF concentrations on the percentage of cryopreserved linear motile spermatozoa from bull is presented in Fig. 2A. A higher percentage of linear motile spermatozoa was found after 30 min of incubation of thawed semen treated with different concentrations of exogeneous PAF. Statistically significant differences were recorded in minute 90 of incubation.

Fig. 2B presents changes in the percentage of nonlinear motile spermatozoa. Regardless of the incubation time, all samples of semen treated with PAF contained a higher percentage of nonlinear motile spermatozoa. As in the case of linear motility, statistically significant differences were observed in the final period of the experiment.

Changes in the percentage of sperm with plasma membrane integrity are presented in Fig. 3A. Throughout the experiment, regardless of the PAF concentration, semen incubated with the phospholipid contained a higher percentage of spermatozoa with plasma membrane integrity. However, no statistically significant differences were found for this parameter. A similar trend was observed for the percentage of spermatozoa with a high mitochondrial transmembrane potential (Fig. 3B). No statistically significant differences were found for this parameter, either. 

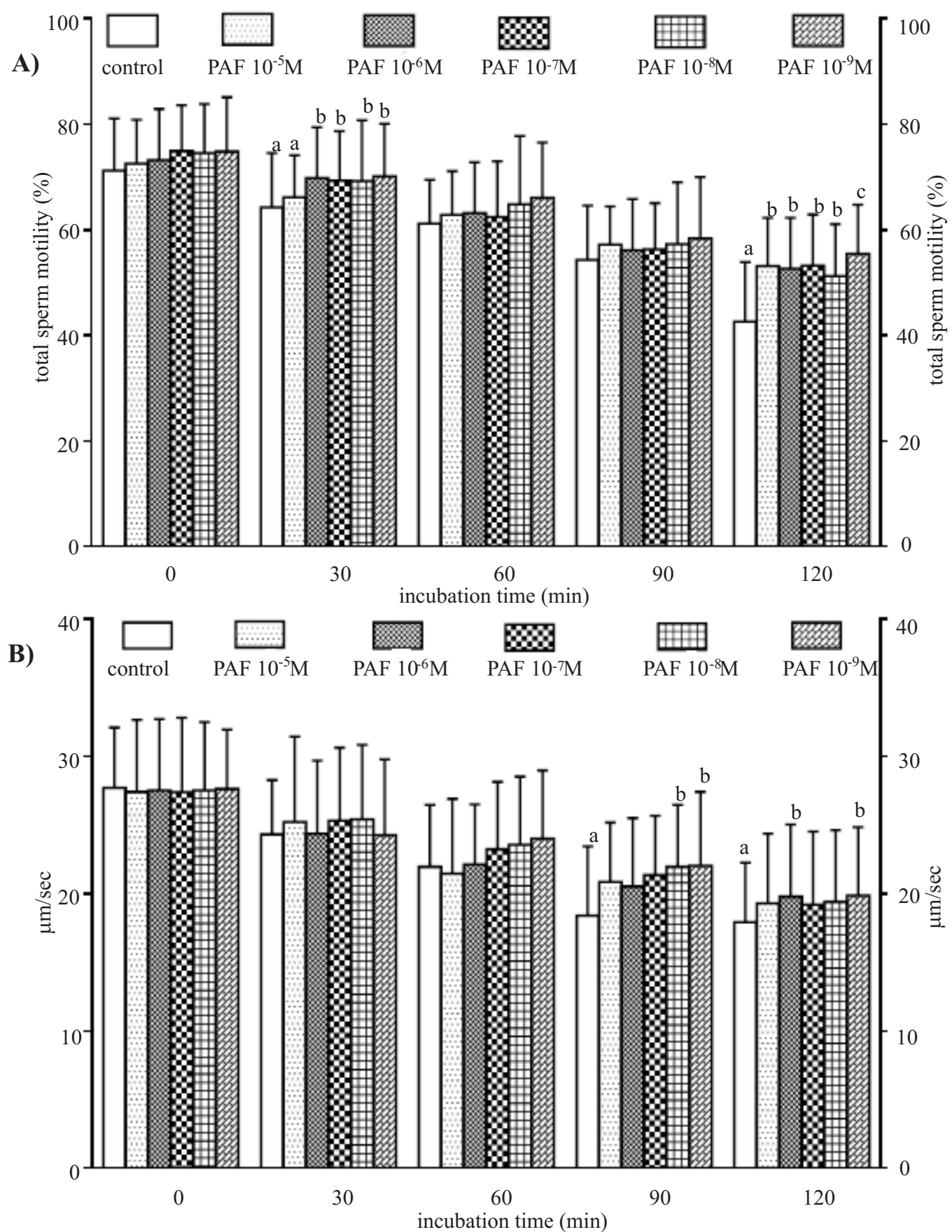

Fig. 1. Percentage of total motility of bull spermatozoa (A) and values of VSL (Straight Line Velocity) (B) following treatment of cryopreserved semen with different concentrations of platelet-activating factor (PAF). Sperm motility was assessed by the computer-assisted semen analysis (CASA) system. Values represent the means \pm SD from 10 bulls. Within incubation time, values $(\mathrm{a}, \mathrm{b}, \mathrm{c})$ with different letters are significant at $\mathrm{p} \leq 0.05$. 

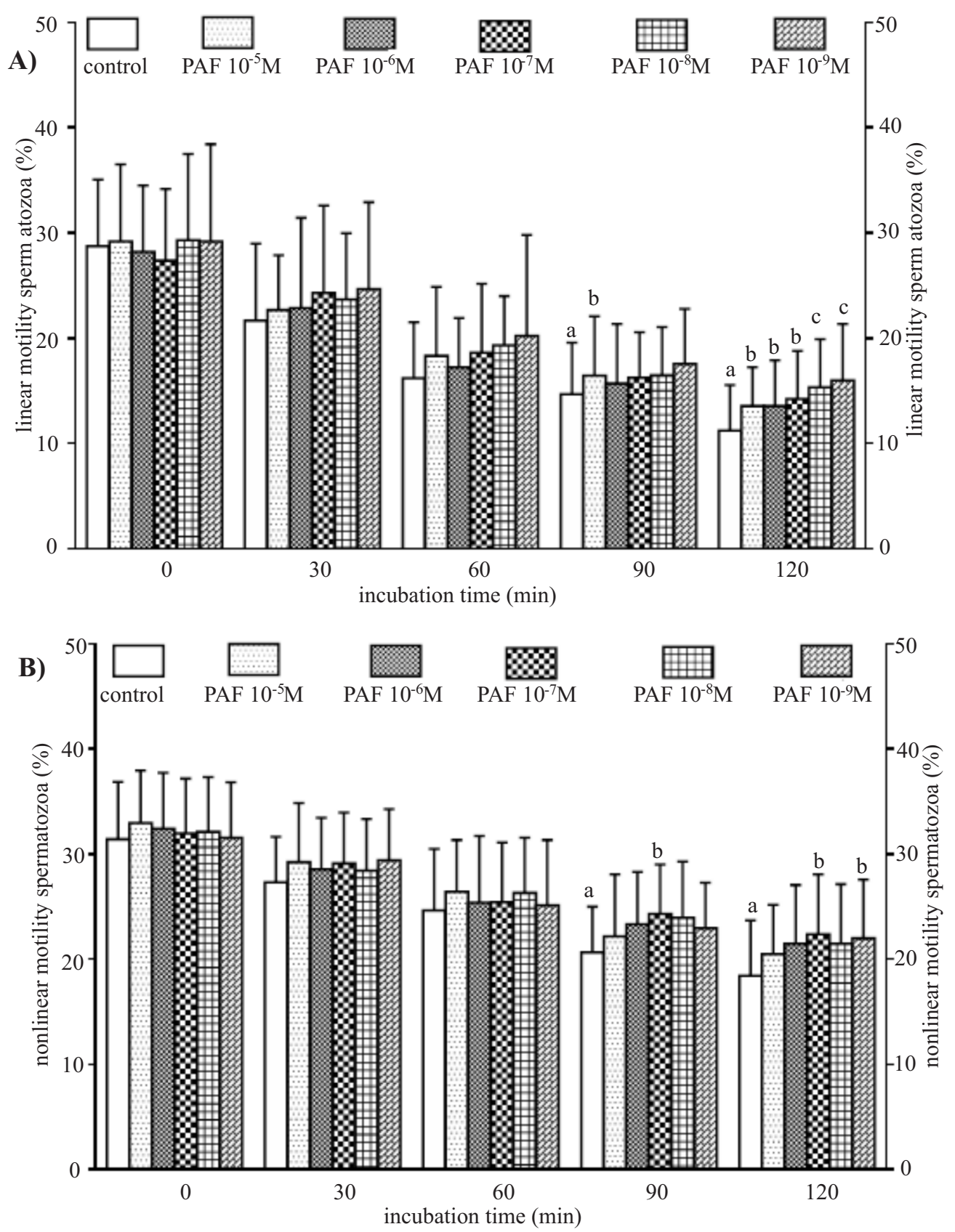

Fig. 2. Percentage of linear (A) and nonlinear (B) bull spermatozoa following treatment of cryopreserved semen with different concentrations of platelet-activating factor (PAF). Sperm motility patterns were analysed by the computer-assisted semen analysis (CASA) system. Values represent the means \pm SD from 10 bulls. Within incubation time, values (a,b,c) with different letters are significant at $\mathrm{p} \leq 0.05$. 


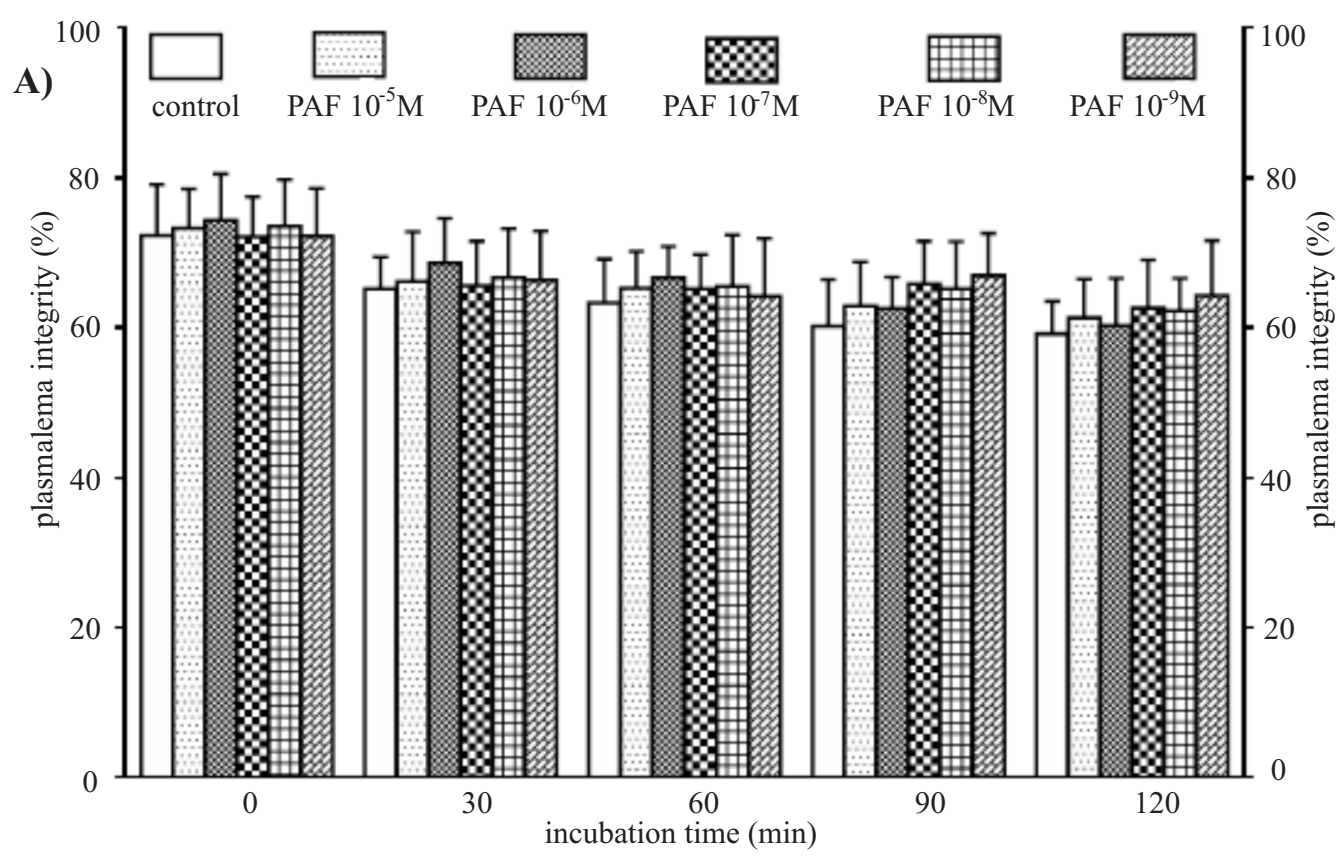

B)

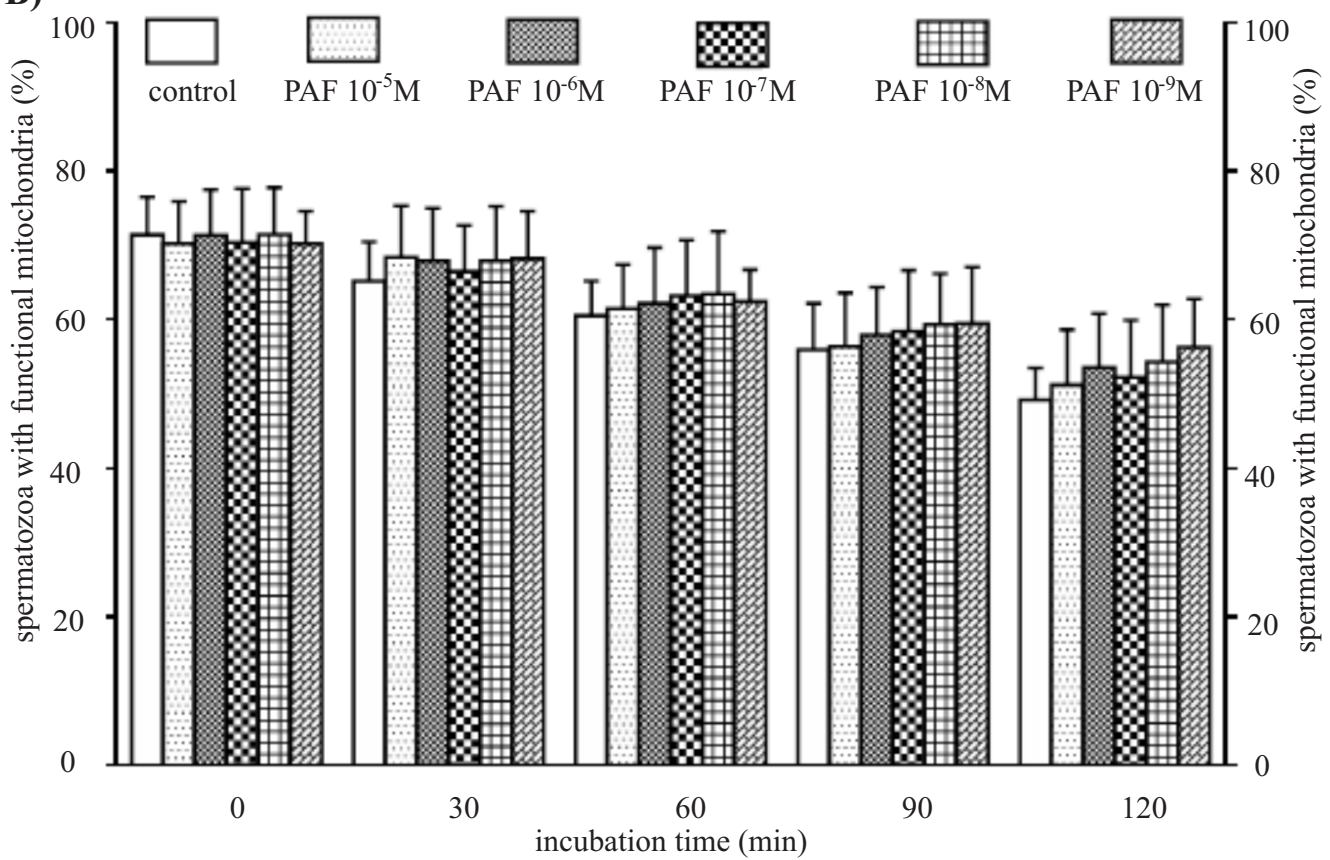

Fig. 3. Percentage of plasma membrane integrity assessed with the SYBR-14/PI assay (A), mitochondrial function assessed with the JC-1/PI assay (B) of bull spermatozoa following treatment of cryopreserved semen with different concentrations of platelet-activating factor (PAF). Values represent the means \pm SD from 10 bulls.

\section{Experiment 2}

Results from experiment 1 demonstrated that $1 \times 10^{-9} \mathrm{M}$ PAF was the optimal concentration, and it had the most beneficial effect on the analysed quality parameters of the cryopreserved bull semen. After se- lecting the optimal PAF concentration, its effect on the cryopreserved bull semen with reduced motility was investigated. Experiment II was carried out on semen samples in which the percentage of motile sperm was below $70 \%$.

Results from the analysis of sperm motility are 

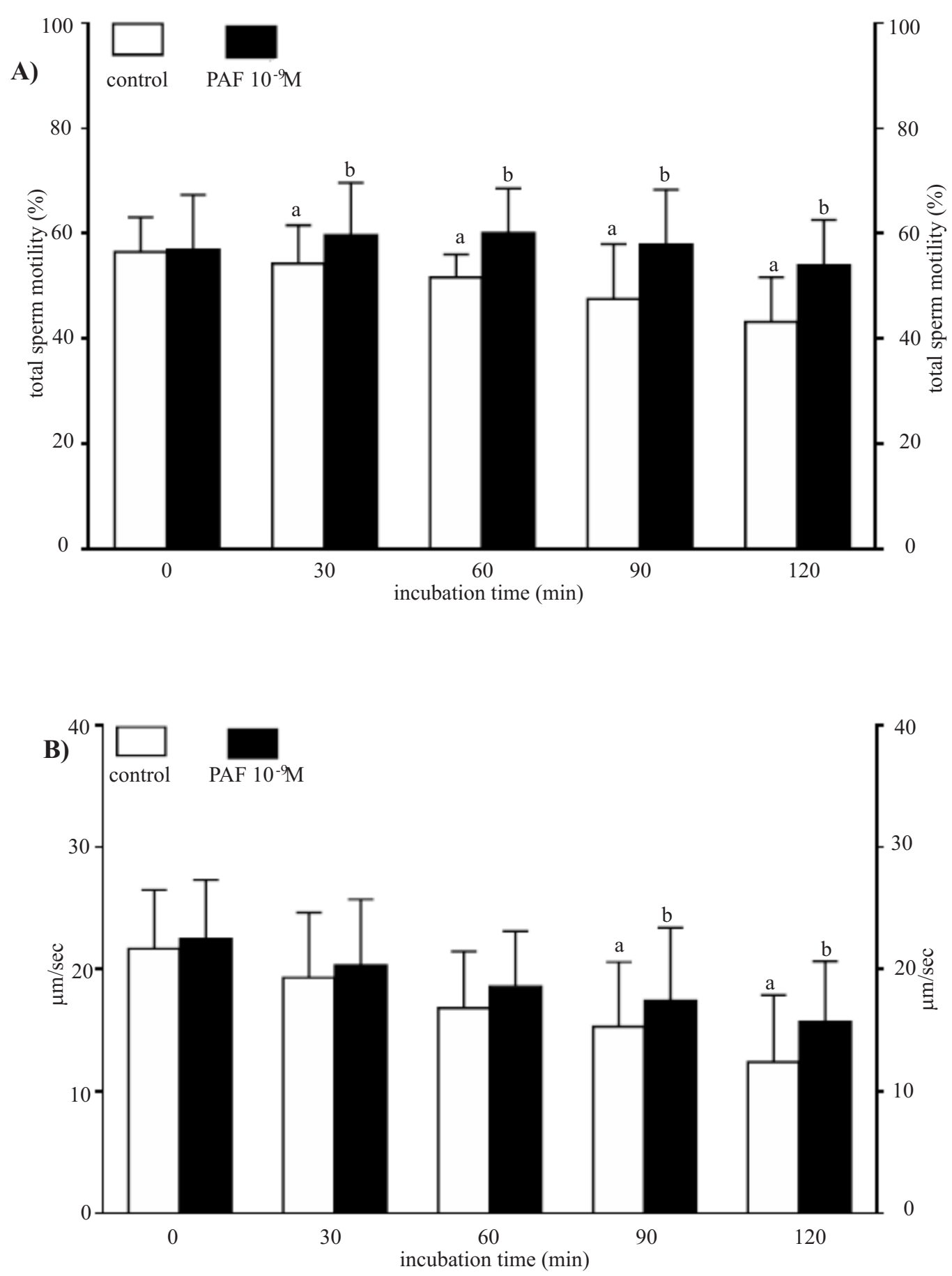

Fig. 4. Percentage of total motility of cryopreserved bull spermatozoa (A) and values of VSL (Straight Line Velocity) (B) for semen samples with reduced motility treated with $10^{-9} \mathrm{M}$ exogeneous PAF. Sperm motility was assessed by the computer-assisted semen analysis (CASA) system. Values represent the means \pm SD from 10 bulls. Within incubation time, values (a,b) with different letters are significant at $\mathrm{p} \leq 0.05$.

presented in Fig. 4A. The mean sperm motility in the control sample was $56.5 \%$. From minute 30 statistically significant differences $(\mathrm{p} \leq 0.05)$ in the percentage of motile sperm $(59.69 \%)$ were found between the control and samples treated with $1 \times 10^{-9} \mathrm{M}$ PAF.

Treatment of samples with PAF had positive effects on all kinematic parameters of sperm, i.e. VCL,
VAP, ALH, BCF (data not shown) and VSL. It was found that in minute 90 of the experiment the supplementation with PAF at optimal concentration caused a statistically significant increase in the VSL values of spermatozoa (Fig. 4B).

The effect of the optimal PAF concentration on the percentage of cryopreserved linear motile bull 

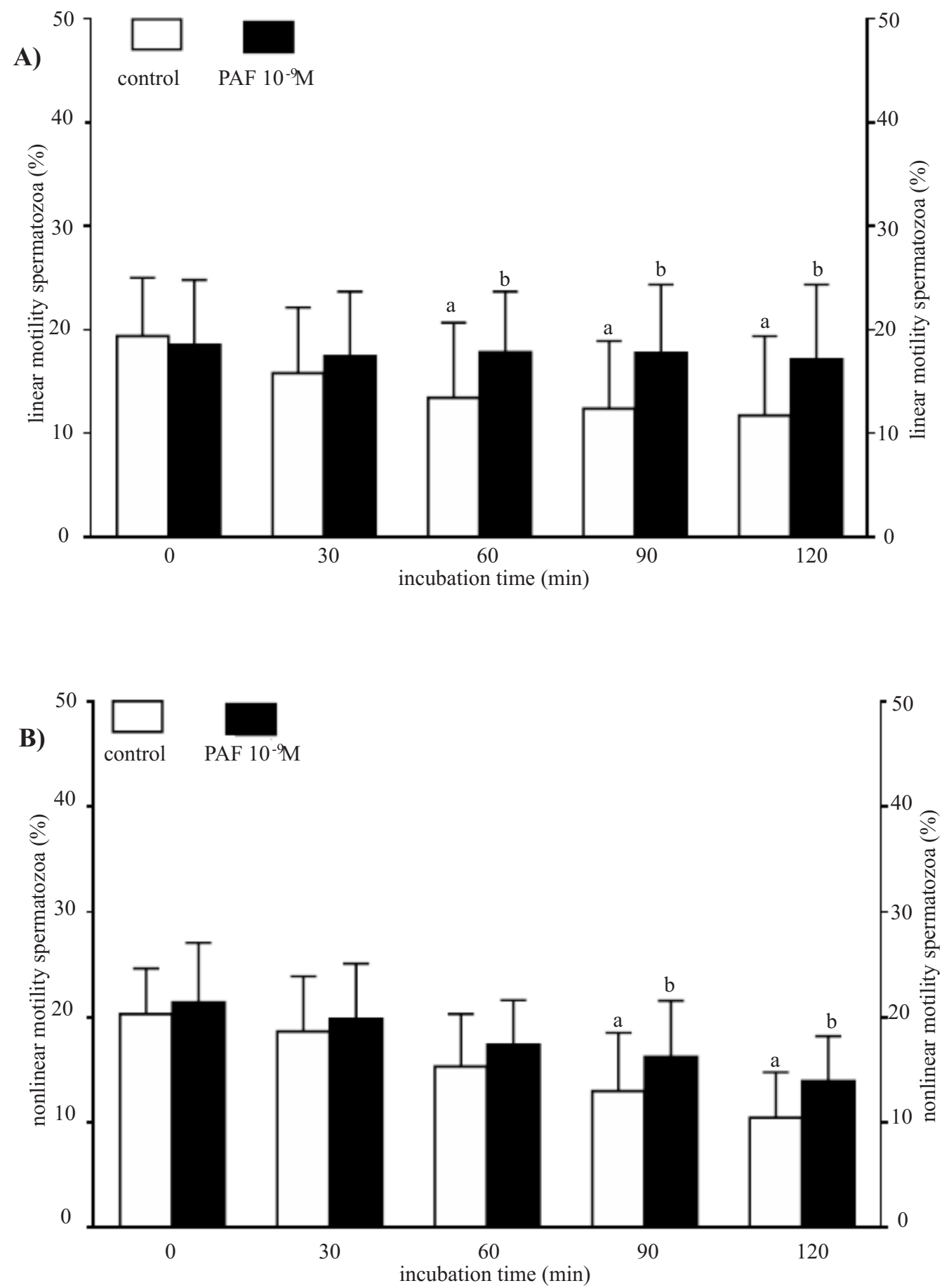

Fig. 5. Percentage of linear (A) and nonlinear (B) of cryopreserved bull spermatozoa in semen samples with reduced motility treated with 10-9M exogenous PAF. Sperm motility patterns were analysed by the computer-assisted semen analysis (CASA) system. Values represent the means \pm SD from 10 bulls. Within incubation time, values $(\mathrm{a}, \mathrm{b})$ with different letters are significant at $\mathrm{p} \leq 0.05$.

spermatozoa is presented in Fig. 5A. An increase in the percentage of linear motile spermatozoa was observed throughout the entire incubation period. Statistically significant differences in this parameter were observed as early as in minute 30 of semen incubation. A similar trend was observed for nonlinear motile spermatozoa (Fig. 5B). An increase in the percentage of nonlinear motile spermatozoa was observed from the first minutes of sample incubation with PAF. In the final period of incubation a statistically significant effect $(\mathrm{p} \leq 0.05)$ of supplementation with PAF on the percentage of nonlinear motile spermatozoa was found.

Changes in the percentage of sperm with the inte- 

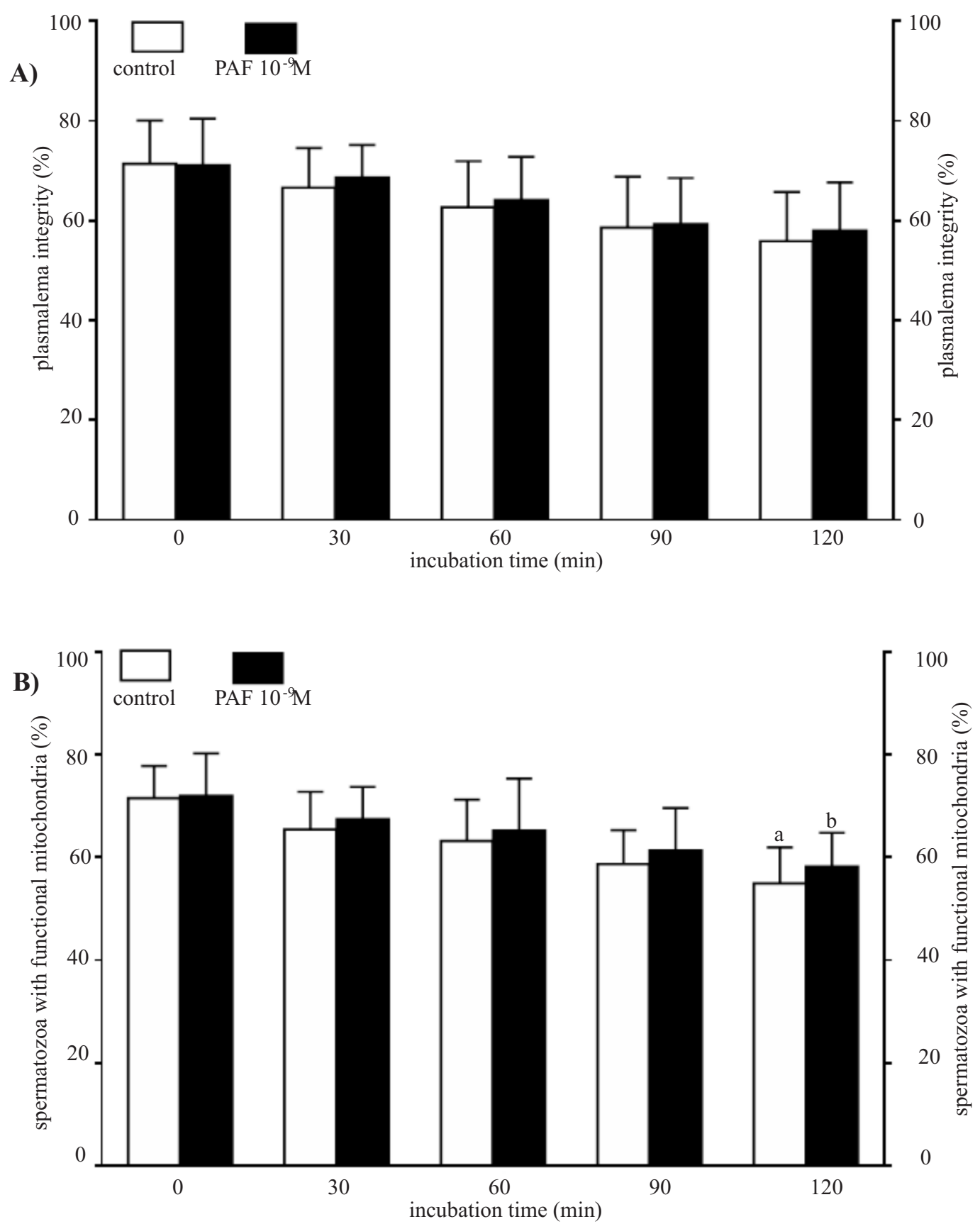

Fig. 6. Percentage of plasma membrane integrity assessed with the SYBR-14/PI assay (A), mitochondrial function assessed with the JC-1/PI assay (B) of cryopreserved bull semen samples with reduced motility treated with $10^{-9} \mathrm{M}$ exogenous PAF. Values represent the means \pm SD from 10 bulls. Within incubation time, values (a,b) with different letters are significant at $\mathrm{p} \leq 0.05$.

gral plasma membrane are presented in Fig. 6A. Throughout the experiment, treatment of samples with the optimal concentration of PAF caused an increase in the percentage of sperm with the integral plasma membrane. However, the differences were not statistically significant. Moreover, throughout the entire experiment semen incubated with phospholipid contained a higher percentage of sperm with functional mitochondria (Fig. 6B). Statistically significant differences in this parameter at $p \leq 0.05$ were observed after 120 min of incubation.

\section{Discussion}

The aim of the first experiment was to select the optimal concentration of exogenous PAF that would have a positive effect on the basic parameters of cryopreserved bull semen after thawing. The study revealed that sperm incubated with $1 \times 10^{-9} \mathrm{M}$ PAF contained the highest percentage of motile spermatozoa at all time intervals of the experiment. Moreover, an increase in sperm kinematic parameters, i.e. VCL, VAP, ALH, BCF (data not shown) and VSL, as well 
as in the percentage of linear and nonlinear motile spermatozoa was observed. These results are in line with observations by Ricker et al. (1989), who demonstrated that treatment of human semen for 5 min with synthetic PAF at concentrations from $10^{-7}$ to $10^{-13} \mathrm{M}$ resulted in a significant increase in motility, whereas treatment with $10^{-5} \mathrm{M}$ PAF resulted in immediate cell death. A threefold improvement in the motility of human spermatozoa was reported after exposure to $10 \times 10^{-9} \mathrm{M}$ exogenous PAF for $4 \mathrm{~h}$ (Krausz et al. 1994). Improved motility of stallion sperm exposed to PAF at concentrations from $10^{-10}$ to $10^{-13} \mathrm{M}$ was reported, with the best motility maintained after $120 \mathrm{~min}$ of incubation (Odeh et al. 2003).

On the other hand, Kheradmand et al. (2009) reported that a 30-minute incubation of sperm with $10 \times 10^{-9} \mathrm{M}$ PAF initiates the acrosome reaction in the ram without a drastic decrease in sperm motility. The effect of exogenous PAF on the biological parameters of human and animal semen can be explained by the function of this endogenous phospholipid. Studies by Reinhardt et al. (1999) and Roudebush et al. (2000) demonstrated the presence of PAF receptors on the surface of the plasma membranes of human sperm. The highest number of receptors was found at the connecting piece and midpiece of the spermatozoon, which is extremely important because of the functions performed by these structures. The midpiece of the spermatozoon, a structure rich in mitochondria, is involved in maintaining sperm motility, while the connecting piece contains proximally located centrioles, participating in the pre-implantation development of the embryo (Santhanthan et al. 1996).

Binding PAF to its receptor protein on the surface of the sperm plasma membrane activates the phospholipase $\mathrm{C}$ system, causing the release of the second messengers in the cell - phosphatidylinositol derivatives - responsible for the increase in the intracellular levels of $\mathrm{Ca}^{2+}$ (Lapetina et al. 1982). All these events increase actin membrane network depolymerisation and phospholipase activation, both of which can increase cellular movement and sperm motility (Roudebush et al. 2000). Studies carried out by Roudebush et al. (2004) demonstrated that damaged human spermatozoa have a modified distribution of PAF receptors on their surface. Abnormal distribution of PAF receptors is associated with disturbed expression of the gene responsible for the synthesis of PAF (Roudebush et al. 2000).

The aim of the second experiment was to assess the effects of the optimal concentration of exogenous PAF on the samples of cryopreserved and thawed bull semen, containing less than $70 \%$ of motile sperm, which in practice do not meet the criteria required by insemination centres. Sperm motility analysis demon- strated a statistically significant increase in the percentage of motile sperm in samples incubated with the optimal concentration of exogenous PAF throughout the entire experiment. Similar results were obtained when analysing the percentage of cryopreserved linear and nonlinear motile spermatozoa. Of all the analysed kinematic parameters of sperm with reduced motility, the addition of the phospholipid caused a statistically significant increase in VSL. Antończyk et al. (2010) indicated a strong correlation between the kinematic parameters of sperm, such as VSL, VCL and ALH and fertility. Similar findings on sperm motility and its basic kinematic parameters were made by Grassi et al. (2010) in their study on human sperm with reduced motility intended for intra-uterine insemination. The researchers reported that a 60 min exposure of sperm to $0.5 \mu \mathrm{M}$ PAF caused a significant increase in the motility parameters. Studies by Briton-Jones et al. (2001) demonstrated that incubation of cryopreserved oligospermic human semen with exogenous PAF increased the percentage of motile spermatozoa. In addition, the exposure of semen samples from patients diagnosed with acute asthenozoospermia to PAF significantly improved motility, but also facilitated the identification of viable sperm necessary for the intracytoplasmic sperm injection (ICSI).

The plasma membrane is responsible for intracellular homeostasis necessary to preserve the in vitro fertilizing capacity of previously preserved sperm (de Andrade et al. 2007). Both the first and the second part of the experiment revealed the positive effects of PAF on the sperm plasma membrane and mitochondrial activity. Similar results were obtained in our previous studies investigating the effect of PAF supplementation on the plasma membranes of cryopreserved boar and canine spermatozoa (Kordan et al. 2009, Kordan et al. 2010). Sperm with a stable plasma membrane have a high transmembrane potential (Trzcińska et al. 2008), which is necessary to generate energy in the form of ATP to maintain sperm motility and other processes (de Andrade et al. 2007). Considering the specific distribution of PAF receptors in the structure of the sperm, it can be assumed that its biological function is also related to the protection of the plasma membrane and sperm motility due to the effect on the mitochondrial transmembrane potential.

In conclusion, the treatment of cryopreserved bull semen characterised by reduced motility (below 70\%) with $10^{-9} \mathrm{M}$ exogenous PAF has a positive effect on semen quality parameters. This is manifested by increased sperm motility, and also improved kinematic parameters and increased stability of the plasma membrane, which is important for insemination practice.

This work received financial support from the $\mathrm{Na}$ - 
tional Centre of Scientific Research, grant no. N N311 524940, and from the University of Warmia and Mazury in Olsztyn (no. 11.610.003-300).

\section{References}

Antończyk A, Niżański W, Twardoń J, Kozdrowski R, Ochota M, Błasiak K, Mikołajewska N, Stańczyk E (2010) Current views on computer assisted sperm analysis. Med Weter 66: 663-667.

Aravindakshan TV, Sharma A (1996) Effect of platelet activating factor on the motility and acrosome reaction of buffalo (Bubalus bubalis ) spermatozoa. Theriogenology 45: 991-999.

Braquet P, Touqui L, Shen TY, Vargaftig BB (1987) Perspectives in platelet-activating factor research. Pharmacol Rev 39: 97-145.

Briton-Jones C, Yeung QS, Tjer GC, Chiu TT, Cheung LP, Yim SF, Lok IH, Haines C (2001) The effects of follicular fluid and platelet-activating factor on motion characteristics of poor-quality cryopreserved human sperm. J Assist Reprod Genet 18: 165-170.

de Andrade AF, de Arruda RP, Celeghini EC, Nascimento J, Martins SM, Raphael CF, Moretti AS (2007) Fluorescent stain method for the simultaneous determination of mitochondrial potential and integrity of plasma and acrosomal membranes in boar sperm. Reprod Domest Anim 42: 190-194.

Diaz E, Szeto AC, Roudebush WE (1999) Presence of platelet-activating factor in rhesus (Macaca mulatta) spermatozoa. J Med Primatol 28: 32-35.

Dziekońska A, Fraser L, Strzeżek J (2009) Effect of different storage temperatures on the metabolic activity of spermatozoa following liquid storage of boar semen. J Anim Feed Sci 18: 638-649.

Esmaeilpour T, Zarei M-R, Bahmanpour S, Aliabadi E, Hosseini A, Jaberipour M (2014) Effect of follicular fluid and platelet-activating factor on lactate dehydrogenase $\mathrm{C}$ expression in human asthenozoospermic samples. Iran J Med Sci 39: 20-28.

Elia J, Imbrogno N, Delfino M, Mazzilli R., Rossi T, Mazzilli F (2010) The importance of the sperm motility classes - future directions. The Open Journal of Andrology 2: $42-43$.

Estienne MJ, Harper AF, Day JL (2007) Characteristics of sperm motility in boar semen diluted in different extenders and stored for seven days at $18^{\circ} \mathrm{C}$. Reprod Biol 7: 221-231.

Garner DL, Johnson LA (1995) Viability assessment of mammalian sperm using SYBR-14 and propidium iodide. Biol Reprod 53: 276-284.

Grassi G, Cappello N, Gheorghe MF, Salton L, Di Bisceglie C, Manieri C, Benedetto C (2010) Exogenous platelet-activating factor improves the motility of human spermatozoa evaluated with C. A. S. A.: optimal concentration and incubation time J Endocrinol Invest 33: 684-690.

Harper MJK (1989) Platelet-activating factor: a paracrine factor in preimplantation stages of reproducion? Biol Reprod 40: 907-913.

Hough SR, Parks JE (1994) Platelet-activating factor acetylhydrolase activity in seminal plasma from the bull, stallion, rabbit, and rooster. Biol Reprod 50: 912-916
Johnson LA, Weitze KF, Fiser P, Maxwell WM (2000) Storage of boar semen. Anim Reprod Sci 62: 143-172.

Kaeoket K, Srisowanna T, Wichaidit U, Chanapiwat P, Manee-in S (2010) Comparative study on six different long term commercial extenders for fresh boar semen. Thai J Vet Med 40: 257-263.

Kheradmand A, Sookhtezary A, Moosavi SM, Joorabi S (2009) The effect of platelet activating factor on the motility and acrosome reaction of ram spermatozoa. Iran J Vet Res 10: 315-322.

Kordan W, Lecewicz M, Strzeżek R, Dziekońska A, Fraser $\mathrm{L}$ (2010) Effect of platelet activating factor (PAF) supplementation in semen extender on viabilty and ATP content of cryopreserved canine spermatozoa. Pol J Vet Sci 13: 571-579.

Kordan W, Lecewicz M, Tobolski J (2009) Effect of platelet-activating factor on motility, plasmalemma integrity, the process of capacitation and acrosome reaction of fresh and cryopreserved boar spermatozoa. Pol J Vet Sci 12: $175-181$.

Kordan W, Strzeżek J (2006) Synthetic palatelet activating factor (PAF) as a boar semen extender component. Med Weter 62: 560-562.

Kordan W, Strzeżek J, Fraser L (2003) Functions of platelet activating factor (PAF) in mammalian reproductive processes: a review. Pol J Vet Sci 6: 55-60.

Krausz C, Gervasi G, Forti G, Baldi E (1994) Effect of platelet-activating factor on motility and acrosome reaction of human spermatozoa. Hum Reprod 9: 471-476.

Kumar R, Harper MJ, Hanahan DJ (1988) Occurrence of platelet-activating factor in rabbit spermatozoa. Arch Biochem Biophys 260: 497-502.

Lapetina EG (1982) Platelet-activating factor stimulates the phosphatidylinositol cycle. Appearance of phosphatidic acid is associated with the release of serotonin in horse platelets. J Biol Chem 257: 7314-7317.

Minhas BS, Kumar R, Ricker DD, Robertson JL, Dodson MG (1991) The presence of platelet activating factor-like activity in human spermatozoa. Fertil Steril 55: 372-376.

Odeh AI, Dascanio JJ, Caceci T, Bowen J, Eng LA (2003) Effect of platelet-activating factor (PAF) on stallion sperm motility, capacitation and the acrosome reaction. Reproduction 126: 605-613.

Reinhardt JC, Cui X, Roudebush WE (1999) Immunofluorescent evidence of the platelet- activating factor receptor on human spermatozoa. Fertil Steril 71: 941-942.

Ricker DD, Minhas BS, Kumar R, Robertson JL, Dodson MG (1989) The effects of platelet-activating factor on the motility of human spermatozoa. Fertil Steril 52: 655-658.

Roudebush WE, Diehl JR (2001) Platelet-activating factor content in boar spermatozoa correlates with fertility. Theriogenology 55: 1633-1638.

Roudebush WE, Mathur RS (1998) Presence of platelet-activating factor in squirrel monkey (Saimiri boliviensis) spermatozoa: seasonal differences. Am J Primatol 45: 301-305.

Roudebush WE, Wild MD, Maguire EH (2000) Expression of the platelet-activating factor receptor in human spermatozoa: differences in messenger ribonucleic acid content and protein distribution between normal and abnormal spermatozoa. Fertil Steril 73: 967-971. 
Roudebush WE, Gerald MS, Cano JA, Lussier ID, Westergaard G, Higley JD (2002) Relationship between platelet-activating factor concentration in rhesus monkey (Macaca mulatta) spermatozoa and sperm motility. Am J Primatol 56: 1-7.

Roudebush WE, Toledo AA, Kort HI, Mitchell-Leef D, Elsner CW, Massey JB (2004) Platelet-activating factor significantly enhances intrauterine insemination pregnancy rates in non-male factor infertility. Fertil Steril 82: 52-56.

Sathananthan AH, Ratnam SS, Ng SC, Tarin JJ, Gianaroli A, Trounson A (1996) The sperm centriole: its inheritance, replication and perpetuation in early human embryos. Hum Reprod 11: 345-356.
Thomas CA, Garner DL, DeJarnette JM, Marshall CE (1998) Effect of cryopreservation on bovine sperm organelle function and viability as determined by flow cytometry. Biol Reprod 58: 786-793.

Trzcińska M, Bryła M, Smorąg Z (2008) Effect of liquid storage on membrane integrity and mitochondrial activity: a new diagnostic method of evaluating boar sperm quality. J Anim Feed Sci 17: 372-380.

Walsh SW, Williams EJ, Evans AC (2011) A review of the causes of poor fertility in high milk producing dairy cows. Anim Reprod Sci 123: 127-138. 